\title{
Las funciones de la educación física escolar: una mirada centrada en la justicia social y la reconstrucción del conocimiento*
}

\author{
Physical Education functions in school: an approach focused on social justice and \\ knowledge reconstruction
Funções da Educação Física escolar: uma análise centrada na justiça social e na reconstrução do conhecimento

\section{Alberto Moreno Doña ${ }^{a}$, Marlen Campos Vidal ${ }^{b}$, Alejandro Almonacid Fierro $^{c}$}

aUniversidad Austral de Chile, Instituto de Filosofía y Estudios Educacionales, Facultad de Filosofía y Humanidades. Campus Isla Teja S/N, Valdivia, Chile. Telf.: 56-63-293879.

Correo electrónico: amorenodona@gmail.com

bInstituto de Filosofía y Estudios Educacionales, Facultad de Filosofía y Humanidades. Campus Isla Teja

S/N, Valdivia, Chile. Telf.: 56-63-293879. Correo electrónico: marlen.camposvidal@ gmail.com

'Universidad Autónoma de Chile, Facultad de Educación. 5 Poniente, 1670. Talca, Chile. Telf.: 6-71-342832.

Correo electrónico: aalmonacidf@uautonoma.cl

\begin{abstract}
RESUMEN
El trabajo que a continuación se presenta es un intento por sintetizar las diferentes miradas y perspectivas epistemológicas y profesionales de la Educación Física como asignatura del curriculum escolar. A partir de ello realizamos un posicionamiento teórico en relación a las funciones que, a nuestros entender, debiera cumplir (o estar cumpliendo) la Educación Física en las diferentes instituciones escolares, a saber: un trabajo educativo orientado hacia la consecución de mayores cuotas de justicial social y focalizado en la reconstrucción del conocimiento por parte de los niños y adolescentes, procedentes de la experiencia paralela y anterior a la vida en la escuela.
\end{abstract}

Palabras clave: paradigmas educativos, funciones educación física, justicia social, reconstrucción del conocimiento.

\begin{abstract}
The following study is an attempt to summarize the different epistemological and professional approaches and perspectives of Physical Education as a subject in the schools curriculum. With this study, we defend the following theory in relation to the functions we think Physical Education should have in the different school institutions: it should be an educational work with an orientation towards reaching higher levels of social justice and it should also focus on children and teenagers' knowledge reconstruction (based on parallel and previous experiences) during school ages.

Key words: educational paradigms, physical Education functions, social justice, knowledge reconstruction.

\section{RESUMO}

Intenta-se a síntese dos vários olhares e perspectivas epistemológicas e profissionais da Educação Física como disciplina do currículo escolar. A partir disso, posiciona-se teoricamente em relação às funções que, ao nosso ver, a Educação Física nas diferentes instituições de ensino deveria cumprir (ou estar cumprindo), quais sejam: um trabalho educativo voltado para o alcance de níveis mais elevados de justicia social e focado na reconstrução do conhecimento procedente de experiência paralela e anterior à vida na escola, por parte de crianças e adolescentes.
\end{abstract}

Palavras chave: paradigmas educacionais, funções da Educação Física, justiça social, reconstrução do conhecimento.

* Este trabajo surge en el contexto, y con el patrocinio y apoyo del Proyecto de Investigación N N 11110016 , financiado por el Fondo Nacional de Investigación Científica y Tecnológica de Chile (Fondecyt). 


\section{INTRODUCCIÓN}

Las corrientes que dan sustento epistemológico a las diferentes asignaturas del curriculum escolar, en general, y a la educación física, en particular, son construcciones sociales que se han ido construyendo, en el tiempo, a partir de los conocimientos, creencias, formas de pensar y de sentir en relación a esa área de estudio. En el caso particular de la educación física escolar, estas construcciones socioculturales representan diferentes maneras de ver y entender el cuerpo y el movimiento. Estas formas de entender el cuerpo y el movimiento, como eje central de la asignatura, provocan que se tenga posiciones encontradas y, como consecuencia de ello, diferentes percepciones en relación a cuáles deberían ser aquellos aspectos de la cultura que necesitan ser transmitidos a partir de la educación física escolar, intentando excluir aquellas otras concepciones que pudieran provocar otra selección cultural diferente.

En el caso concreto de nuestra asignatura, la situación es bien especial, pues, aunque históricamente han existido y existen diferentes corrientes y enfoques del área, éstos no se han excluido unos a las otros sino que se han ido incluyendo en los currículum escolares, en una constante lucha por tener más poder y mayor presencia en los mismos (Hernández, 1996). El modo en el que la "la educación física ha ido incorporando las nuevas corrientes (mediante procesos de mera yuxtaposición en la que los distintos agregados presentan contradicciones mutuas) genera serias dudas sobre la naturaleza de la propia disciplina" (Barbero, 2007: 36).

\section{PERSPECTIVAS, CORRIENTES Y PARADIGMAS DE LA EDUCACIÓN FÍSICA ESCOLAR}

Según Hernández Álvarez (1996), son cuatro las corrientes actuales de la Educación Física como asignatura del curriculum escolar, a saber:

-Deportes: es aquella corriente a partir de la cual se hace un traslado, casi mecánico, de los principios y procedimientos de la lógica del deporte de élite a la estructura de la educación física escolar, siendo la competición deportiva y la victoria en la misma el centro de las prácticas escolares para buscar el prestigio institucional y personal.

-Condición Física: la condición física también ha intentado convertirse en el centro hegemónico a partir del cual gire la educación física escolar. Según el autor en el que nos estamos centrando, los antecedentes de esta corriente habría que situarlos en la lógica científica cuya intencionalidad ha estado en crear las bases anatómicas y fisiológicas del movimiento humano. Bajo esta corriente, es posible visualizar una asociación entre "la condición física y una idea reduccionista de la salud, en cuanto a su concepción puramente física y objetiva" (Hernández, 1996: 60).

-Psicomotricidad: es bajo esta corriente donde comienza a pensarse en un cuerpo menos reduccionista que se aleja de lo puramente físico y objetivo. Es el cuerpo pensante el centro de esta tendencia, entendiendo que a través del movimiento corporal es posible el desarrollo intelectual de la persona. Si bien comienza a haber un alejamiento del conocido 'cuerpo máquina' como sustento de la educación física escolar y representado, principalmente, a partir de las dos concepciones anteriores, también es cierto que esta 
corriente estuvo focalizada en las edades más tempranas del niño, por lo que su fuerza en la educación física en general ha sido y es limitada.

-Expresión corporal: se orienta, principalmente, hacia el desarrollo de las capacidades rítmico expresivas del cuerpo que se expresan a través del movimiento. Es la creatividad natural en el ser humano el centro de su existencia, argumentando que los movimientos estereotipados presentes en el deporte de competición no permiten y legitiman la misma.

En el mismo sentido de lo mostrado en relación a las diferentes corrientes existentes en la educación física escolar, aunque con algunas diferencias conceptuales, Vázquez (2001) hace un análisis de las diferentes formas de entender la asignatura a partir de los diferentes paradigmas o formas de concebir el movimiento humano. Así, la autora realiza una clasificación de las diferentes concepciones del movimieno humano que pueden visualizarse en el área. Esta clasificación procede de tres paradigmas diferentes del movimiento humano, a saber:

-Biomotriz

-Psicomotriz

-Expresivo

Estos tres paradigmas estarían sustentados en tres formas diferentes de entender, percibir y conceptualizar el cuerpo humano:

-Cuerpo máquina: objetivización del cuerpo a partir de la metáfora que permitiría entender que el cuerpo se comporta como una máquina perfecta en la cual es posible predecir su comportamiento.

-Cuerpo psicosomático: relación entre cuerpo y mente como dos realidades que funcionan a partir de la información que ambas se transmiten.

-Cuerpo expresivo: el cuerpo como sistema de comunicación.

En el paradigma biomotriz, según Vázquez (2001), el movimiento es el resultado de las estructuras biológicas que le dan sentido, y para mejorarlo habría que ser consecuentes con las leyes de la anatomía, la fisilogía y la biomecánica. Es por ello que una educación física centrada y fundamentada en este paradigma se dirigiría hacia el desarrollo de la condición biológica para conseguir un mejor y mayor rendimiento y eficiencia motriz. El desarrollo de la fuerza, la flexibilidad, resistencia, velocidad y agilidad sería sus objetivos, y los juegos, los deportes y la gimnástica serían sus contenidos.

Es interesante que Vázquez (2001) difiera de la clasificación realizada por Hernández Álvarez (1996), pues para la primera el deporte y la condición física formarían parte de una misma corriente (o paradigma) mientras que para el segundo se hace más conveniente realizar tal separación, pues la "existencia de poblaciones específicas que precisan del desarrollo de una buena condición física en sus labores profesionales, así como la población en general que persigue metas de salud, hacen posible su separación del deporte y la configuración de una corriete propia" (Hernández, 1996:60).

El paradigma psicomotriz, como señala Vázquez (2001), nace como consecuencia de la consideración de otras disciplinas científicas al estudio y aplicación del cuerpo humano diferentes a la anatomía, fisiología y biomecánica, como son las disciplinas más relacionadas con las ciencias neurológicas, las de la conducta y la sociales. Esta visión paradigmática se aleja de la metáfora del cuerpo máquina anclado en el dualismo jerárquico cuerpo-mente y se adentra en la noción de un cuerpo subjetivo que es el que 
uno vive y experimenta, a la vez que el movimiento "es una respuesta tanto a estímulos internos como externos (...) y la regulación del mismo es siempre interna y ésta es lo que le da su carácter personal y no estandarizado" (Vázquez, 2001: 61).

Bajo esta visión paradigmática los objetivos de la educación física escolar se centran, fundamentalmente, en el desarrollo de los diferentes factores psicomotores, como son la coordinación, equilibrio, lateralización, estructuración espacio-temporal, entre otros. En cuanto a los contenidos de enseñanza, estos son variados y múltiples; en ellos lo que prevalece es una serie de estrategias metodológicas de carácter abierto y activo, gracias a las que se genera la organización de diferentes experiencias motrices en función de las cuales los estudiantes descubren, no sólo a partir de los componentes motores, sino también a partir de la inteligencia y la imaginación.

Para Vázquez (2001), el paradigma expresivo se centra en la dimensión comunitiva del ser humano, aspecto excluido, casi en su totalidad, del paradigma biomotriz preponderante en el área. Este paradigma se aleja de la consideración instrumental del movimiento y se focaliza en el carácter expresivo del mismo, expresión esta que se considera mediada por el contexto social una vez el sujeto se desarrolla y evoluciona. Es la armonía entre la expresión espontánea y su posterior regulación en el ámbito social uno de los objetivos centrales de la educación física bajo esta visión paradigmática. La idea es entender el movimiento como un instrumento de comunicación, partiendo de diferentes disciplinas científicas como las ciencias de la conducta, la psicología profunda, la semiótica, la kinésica y proxémica, etc. Entender que no existen modelos predeterminados de movimiento, bajo la consideración de que el cuerpo y el movimiento son construcción personales, lleva a esta visión a proponer una enseñanza no directiva y donde lo fundamental es la experiencia personal vivida por los estudiantes. El acento siempre está en el valor expresivo del movimiento y no en la medida, rendimiento y competición del mismo. Es la expresión corporal el contenido central de esta visión, considerando la danza clásica y moderna, el teatro, etc.

Es la misma autora quien argumenta que, independientemente de la diferenciación que podamos llegar a hacer en función de paradigmas o corrientes, la acción motriz humana siempre se presenta como unidad y, por tanto, el movimiento no es a veces psicomotriz y otras expresivo, sino que cualquier movimiento que realicemos es propio de las tres dimensiones. Es desde esta aclaración que Vázquez (2001) pretende adentrarse en la praxeología motriz, preguntándose si ésta se constituye en un nuevo paradigma, pues según ella dicha visión destaca ampliamente la concepcion de unidad característica de la acción que acabamos de comentar.

La praxeología motriz es un intento por acabar con los reduccionismos propios de la educación física escolar y por encontrar un marco teórico propio del área, como así lo propone su teórico más importante, Parlebas. Si bien este autor (Cit. en Vázquez, 2001) asume que la noción de psicomotricidad es la corriente que mejor representa los intentos de acabar con los mecanicismos y los dualismos de la asignatura, también le parece insuficiente para explicar el movimiento humano, pues olvida la dinámica relacional de la motricidad humana, aspecto definido por él como socio-motricidad, haciendo alusión a la dimensión social y comunicativa de la conducta humana.

"La noción de este autor por encontrar un marco científico propio y específico que fundamente las prácticas físicas y, sobre todo, por delimitar el objeto específico de estudio, le lleva a un recorrido epistemológico en el que aporta sucesivamente nuevos materiales (...) $\mathrm{Su}$ 
incomodidad para considerar el movimiento como objeto específico de estudio debido a sus resonancias mecanicistas le lleva a proponer el término conducta motriz, más comprensivo, como el verdadero objeto de la Educación Física (...) y buscar así una ciencia autónoma a la que denomina praxeología motriz" (Vázquez, 2001: 65).

En esta búsqueda incesante de un objeto de estudio propio para la educación física como disciplina científica y disciplina escolar, es donde lleva años trabajando el llamado paradigma de la motricidad humana. Esta visión del área se viene trabajando, principalmente, en Portugal y en un grupo de países latinoamericanos como Colombia, Brasil y Chile. La motricidad humana, como paradigma emergente, surge del análisis de la educación física a partir del aporte de las llamadas ciencias de la complejidad (Sergio, 2009; Trigo y Montoya, 2007; Toro, 2007a, 2007b y 2009).

La intención principal de la ciencia de la motricidad humana es trasladarse desde lo físico al "movimiento intencional de la trascendencia" (Sergio, 2009: 31), la complejidad del ser humano presente en las aulas escolares, atendiendo al cuerpo, la mente, el deseo, la naturaleza y la sociedad. La ciencia de la motricidad humana es "el cuerpo en acto, es virtualidad para la acción, es el movimiento intencional de trascender y trascenderse" (Sergio, 2009: 31).

Una de las ideas principales de la ciencia de la motricidad humana es que la educación física, mientras siga siendo física, seguirá alienando los cuerpos, y como cuerpo y sujeto son inseparables, seguirá alienando a los sujetos, por tanto, no siendo verdadera educación (Trigo y Montoya, 2007). Para que ello no ocurra es importante comenzar considerando que el ser humano no tiene un cuerpo sino que es cuerpo, pues la realidad corporal no es anexa al ser humano sino que es el ser humano en sí mismo.

Según Toro (2007b), mientras nuestra asignatura siga siendo física, seguirá obedeciendo a una racionalidad técnica o instrumental, ocupando los términos de Habermas (1999), por lo que se propone adentrarse en la concepción del hombre como unidad (Morin, 2001). Para ello, centra el objeto de estudio de la educación física (motricidad humana, dirían ellos), no en el cuerpo y el movimiento, sino en la acción humana, pues en la acción humana se despliega toda la complejidad integral del ser humano que la realiza -una acción que en el ámbito educativo-escolar debe ir direccionada hacia el desarrollo humano.

"Este modelo se sustenta en la acción humana y no en el movimiento. Desde este enfoque se entiende al ser humano como un ser que configura mundos desde su accionar, plegado y pleno de sentido, de significados y símbolos, orientado hacia fines y afectos, ilusión e imaginación, proyección y potencia de sí mismo en un contexto comunitario, nunca aislado, pero al mismo tiempo irrenunciablemente convocado a decidir su propia existencia y la de los demás cercanos a él o ella, siempre más o menos consciente de quién es, con quienes está y dónde está" (Toro, 2007a: 24).

La red internacional de investigadores en Motricidad Humana (2006) señala que la ciencia de la Motricidad Humana nace, por tanto, de tres cortes epistemológicos. El primero es el de la disciplina, la educación física; el segundo es el del concepto clásico de ciencia; el tercero se refiere al concepto de conocimiento. En consecuencia, la Motricidad Humana viene a representar una nueva ciencia del ser humano, en la búsqueda permanente de su desarrollo.

Desde la ciencia de la Motricidad Humana, la Educación Física es vista como un espacio generoso en la construcción de sentidos y significados, para que hombres y 
mujeres puedan levantar proyectos de vida a partir de la relación, y en consecuencia, construir y compartir mundos posibles, teniendo como telón de fondo la humanización de las actividades deportivas y recreativas que despliega el ser humano. En definitiva, la Motricidad Humana nos otorga la posibilidad de entender al hombre y a la mujer desde una mirada más humana, más plena, más sistémica y, en consecuencia, enriquece nuestra reflexión otorgando un carácter eminentemente pedagógico y de desarrollo humano al ámbito educativo-deportivo (Trigo, 2006; Sergio 2006; Benjumea, 2009; Jaramillo, 2010).

Así, se propone para la Educación Física, el Deporte y la Recreación humanizar las actividades deportivas, de salud, juego y trabajo que realiza el ser humano, entendiendo el humanismo desde la visión filosófica y hologramática, utilizando y revalorizando las actividades humanas como medio de desarrollo de la conciencia y a partir de ahí ubicarse en el mundo desde una óptica crítica-constructivista; es decir, se trata de entender la educación física, la recreación y el deporte no como movimiento por el movimiento, sino como movimiento con sentido, es decir, cambiar el concepto de desplazamiento de un cuerpo en el espacio, por el concepto de acción (teoría de la acción), como un espacio extremadamente amplio y fecundo de construcción de significados y desarrollo de las formas de pensamiento, aprehensión del entorno y elaboración del mundo propio y compartido.

Paralelamente, se propone despojar de la educación física su función reproductiva, hegemónica de una determinada concepción del deporte y del modelo de sociedad que éste busca y plantea, lo que supone una revisión de la terminología y los supuestos conceptuales de la Educación Física. En pro de una evolución de la misma, el objeto es darle mayor coherencia y pertinencia, acentuando, además, la complejidad de los fenómenos y el anti-dualismo del pensamiento actual (Trigo y Montoya, 2004).

Entendiendo que existirán profesionales del área que podrán estar más de acuerdo con unas u otras de las visiones paradigmáticas presentadas hasta el momento, lo que parece claro es que el desarrollo de todas ellas se ha realizado en una constante lucha contrahegemónica (Barbero, 2007; Hernández, 1996). Es interesante rescatar, aquí, el trabajo de Barbero (2007) desde el concepto de capital corporal, al plantearnos que estas diferentes corrientes de la Educación Física crean y recrean diferentes visiones de nuestra materia, probablemente contradictorias entre sí. Es por ello que se propone visualizar nuevos derroteros que nos permitan imaginar una nueva educación física sustentada en la no separación del yo y el cuerpo que se desarrolla en un contexto social determinado. Para ello Barbero (2007: 21) sugiere realizar un trabajo en conjunto "entre los diferentes especialistas de las cada vez más separadas distintas corrientes".

En esta diversidad de corrientes y visiones paradigmáticas, siendo consciente de la relación de poder que se estabece entre ellas y que la incorporación de las nuevas corrientes ha sido mediante procesos de yuxtaposición -un aparente todo vale-, queremos describir las funciones que se le han atribuido y se le atribuyen a la educación física como asignatura del currículum escolar en las últimas décadas, con el objetivo de relacionarlas.

\section{FUNCIONES DE LA EDUCACIÓN FÍSICA EN EL CONTEXTO ESCOLAR}

Considerando la clasificación que realiza Díaz Lucea (1994) y que confirman Martín (2009) y Romero (s/f), estas funciones, si bien han sido y son atribuidas a la Educación 
Física escolar, no han tenido el mismo peso a lo largo de la historia, y es por ello que las iremos detallando a continuación.

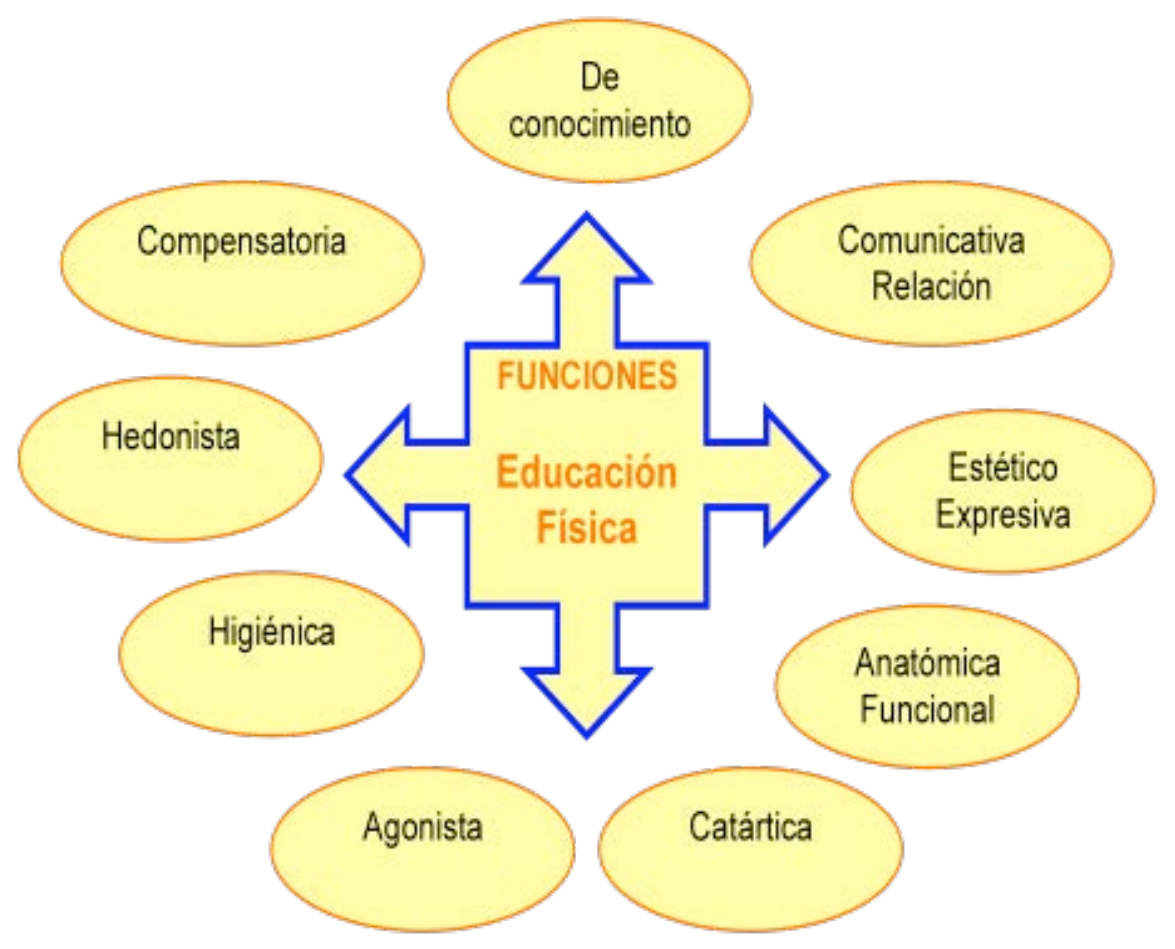

Figura 1. Funciones de la Educación Física Escolar. Fuente: Díaz Lucea (1994)

La función anatómica funcional (FAF) tiene relación con la mejora del desarrollo corporal y de las habilidades y destrezas motrices del sujeto practicante (Martín, 2009; Romero, s/f). Dicho desarrollo puede ser utilizado para variados fines educativos, pero su rol central está en relación con la condición física y la salud en el sistema educativo chileno (Moreno, 2011).

Cuando hablamos de la función higiénica (FH) de la educación física escolar, estamos aludiendo a la conservación y mejora de la propia salud y a la prevención de determinadas enfermedades y disfunciones a través de la actividad física (Martín, 2009; Romero, s/f). Esta función está ligada a la inspiración médica saludable y, por tanto, centra su foco de atención en la excesiva inmovilidad en la escuela y en la sociedad (Toro, 2007b). 
La función estética expresiva (FEE) centra su atención en la imagen corporal y en las manifestaciones artísticas susceptibles de trabajar en el área. Considerando aspectos como el culto al cuerpo, tan presente en la actual sociedad, para el trabajo pedagógico. Según Romero (s/f), "debemos remontarnos a la Grecia clásica, donde se considera una valoración estética hacia el cuerpo, existiendo un culto por la belleza acompañado de virtudes morales e intelectuales, es decir, se perseguía la combinación del hombre de acción con el hombre sabio". No ha sido tan relevante en nuestra área la consideración expresiva, pues no es hasta el siglo XX que comienza a darle valor a la capacidad expresiva del cuerpo a partir de los movimientos.

La función comunicativa y de relación (FCR) focaliza su interés en la capacidad de interaccionar que podemos trabajar en la educación física a través del cuerpo y el movimiento. Esta función fue desarrollada, principalmente, a partir de la Escuela Inglesa con Thomas Arnold, cuando empleaba los juegos y deportes como medios de relación social entre los practicantes.

Por su parte, la función agonística (FA) tiene relación con la competencia física y destreza motriz en su relación con el deseo de competir con otros y consigo mismo. Se dice que fue Pierre de Coubertain, creador de los Juegos Olímpicos modernos el impulsor de esta función (Martín, 2009).

En otra dirección, la función de conocimiento (FCo) se relaciona con el énfasis que los mismos psicomotricistas dieron a la no separación entre inteligencia y movimiento, siendo un aporte importante al área de la educación física, pues el mismo Le Boulch (1969) llegó a plantear que su propuesta no seguía el dualismo de la Educación Física tradicional. Se considera que el cuerpo y el movimiento son elementos centrales en el conocimiento de nosotros mismos y de nuestro entorno. Al considerar la función catártica y hedonista $(\mathrm{FCH})$, estamos relevando las posibilidades del área en relación a convertir la actividad física en una forma de actividad lúdica que ayuda a evadirnos de la realidad y liberarnos de las tensiones cotidianas. Tiene relación con la utilización saludable de los tiempos y espacios de ocio de los que podamos gozar.

Por último, la función de compensación (FC) centra su atención en convertir a la actividad física en un antídoto para los excesivos niveles de sedentarismo presentes en nuestra sociedades y las consecuencias negativas que ello conlleva (Martín, 2009). La idea es poder paliar, desde nuestra área, los efectos negativos de dicho sedentarismo.

Todas estas funciones son recogidas en el sistema educativo chileno (Decreto 220/2005). Para dar cuenta de ello, mostramos un cuadro donde damos a conocer los objetivos de la educación física en el contexto chileno y su relación con las funciones mencionadas.

\section{Objetivos Educación Física en enseñanza media (Chile)}

Ejecutar ejercicios tendientes a incrementar su condición física general, conforme a las posibilidades de avance personal; atender la superación de sus cualidades físicas deficitarias y valorar el ejercicio como factor preventivo que genera hábitos de vida saludables (FAF; $\mathrm{FH} ; \mathrm{FC})$

Apreciar el valor compensatorio y socio-afectivo que genera la realización de actividades al aire libre de carácter recreativo, según las posibilidades de equipamiento y ubicación geográfica de la unidad educativa. (FC; $\mathrm{FCH})$ 
Demostrar control en la combinación de acciones motoras diversas; adaptabilidad perceptiva y decisional al espacio físico, al tiempo y a las interacciones humanas generadas durante la ejecución de tareas motoras específicas (FAF; FCR)

Demostrar progreso en sus indicadores de condición física, conforme a sus posibilidades de superación personal; utilizar procedimientos para controlar y autoevaluar sus propios logros, identificando la relación existente entre salud, calidad de vida y ejercicio físico. (FAF; FH; FCo)

Apreciar el valor compensatorio y socio-afectivo que genera la realización de actividades al aire libre de carácter recreativo, según las posibilidades de equipamiento y ubicación geográfica de la unidad educativa (FC)

Participar activamente, de acuerdo a sus condiciones físicas, en competiciones, eventos y torneos deportivos individuales y de equipo, con y sin oposición, programados por la unidad educativa; apreciar el valor de la participación y la competición deportiva (FA)

Prevenir, al programar y participar en actividades al aire libre y de aventura, los posibles efectos negativos que éstas pueden generar para el medio ambiente en entornos urbanos y naturales (FAF; FCo)

Tabla 1. Objetivos de la Educación Física escolar en Chile. Fuente: elaboración propia

Si observamos la tabla elaborada, podemos percatarnos de que todas las funciones detalladas anteriormente son trabajadas, con mayor o menor presencia, en la educación física chilena.

Ahora bien, creemos que tales funciones no son del todo coherentes con lo que el mismo sistema educativo chileno declara. Es interesante destacar que, en el contexto de la educación física en Chile, se han realizado cambios profundos en la educación física escolar, en donde se establecieron planes y programas, antes inexistentes, y que atienden, "al desarrollo de una actitud crítica y analítica frente a las propias actividades físicas y a la comprensión de los cambios biológicos y psicológicos que los y las jóvenes están experimentando en esta etapa" (MINEDUC, 2010). Todo ello sustentado en una serie de principios básicos que orientan la acción educativa y el quehacer profesional en el sistema educativo, como podemos ver en la siguiente cita.

"La educación debe ofrecer a todos los niños/as y jóvenes la posibilidad de desarrollarse como personas libres, con conciencia de su propia dignidad y como sujetos de derecho. Asimismo, la educación debe contribuir a forjar en ellos el carácter moral regido por el amor, la solidaridad, la tolerancia, la verdad, la justicia, la belleza, el sentido de nacionalidad y el afán de transcendencia personal. El individualismo extremo, que podría resultar de un ejercicio ilimitado de la libertad personal, es moderado por imperativos que brotan de un conjunto de valores que llevan a la persona a compartir con otros los frutos de una libertad que humaniza y se abre a las exigencia del bien común" (MINEDUC, 2009).

Si relacionáramos la funciones de la educación física antes mostradas con las que se reflejan a partir de los objetivos de la educación física en el contexto chileno, podemos percatarnos de que la función más trabajada es la anatómica funcional. Creemos que dicha situación no atiende, en un grado adecuado, a esa actitud crítica supuestamente valorada 
por el Ministerio de Educación (2010). Abogamos, siguiendo a Toro (2007a), que dicha situación parte de una visión de la educación física anclada en el paradigma tecnocrático y muy influenciada por una inspiración médico-saludable y militar (Toro, 2007b). En este mismo sentido, la Educación Física escolar presenta en las aulas de escuelas y liceos del país un fuerte acento en lo deportivo, cuestión que responde a una racionalidad más bien técnica y al discurso físico-deportivo de rendimiento, transmitiendo la idea de que la enseñanza de la Educación Física está basada en el desarrollo de la condición física y las habilidades deportivas (Almonacid y Matus, 2010).

Además, consideramos que la misma clasificación de las funciones de las educación física mostrada responde, aunque explícitamente no lo declara, a una noción del ser humano que posee un cuerpo y no que es un cuerpo, conceptualización esta última ya planteada en el ámbito filosófico por Zubiri (1986). Tal idea implica que la realidad corporal es anexa, lo que nos llevaría a desprendernos de ese algo y quedarnos con la verdadera sustancia del ser humano, el alma, el espíritu o la mente. Además, consideramos que las mismas funciones atienden, poco coherentemente, a las funciones de la escuela como institución social.

Es por ello que nos resulta interesante otra clasificación de las funciones de la educación física, realizada por otros autores (Devís, 1994; Devís y Molina, 1998a; 1998b; 2004; Moreno, 2011) En función de estos autores daremos cuenta, a continuación, de las funciones atribuidas a la educación física tanto en la modernidad como en la postmodernidad, pues nos interesa dar cuenta de este recorrido histórico.

Siguiendo a Devís y Molina (2004), en la modernidad son tres las corrientes que permitieron dar sentido a la educación física y a sus funciones. La primera de ellas es aquella que entendía que la educación física era 'educación de lo físico' y, por tanto, a partir de ella se relevaban los "cuidados, usos y disciplina corporales, la regeneración física y mejora de la raza, la obediencia y la preocupación por la higiene y la salud corporal para hacer ciudadanos productivos y útiles a los Estados modernos". En esta conceptualización podemos percatarnos de la importancia otorgada a la dimensión corporal del ser humano y, sin duda, la explícita separación entre la mente y el cuerpo, postura epistemológica aún presente en muchas de las propuestas actuales del área (Toro, 2007a). Esta dualidad se mantuvo, incluso, en la segunda de las conceptualizaciones presentes en la modernidad en relación a la educación física, aunque es imposible negarse a aceptar el esfuerzo por valorizar la disciplina a partir de considerar que toda actividad motriz, por simple que fuera, llevaba implícita de pensamiento y voluntad para realizarla. Fue así como surge la conceptualización de 'educar a través de lo físico'.

Sin duda que esta conceptualización amplió la mirada, intentando que la educación física fuera algo más allá de lo puramente corporal y físico, pero "seguía orientada a funciones de tipo social de carácter extrínseco y conectadas a los valores dominantes de las sociedades capitalistas" (Devís y Molina, 2004: 38.) Una actividad se valora extrínsecamente cuando se aprecia como un simple instrumento para conseguir unos fines externos a ella misma (Arnold, 1991). Si entendemos la educación física de esta manera, podemos afirmar que tal actividad nos permite desarrollar aspectos concretos de la vida cotidiana de cualquier estudiante, es decir, que es un muy buen instrumento para conseguir numerosos propósitos a desarrollar en el patio escolar. Se está suponiendo que el valor de esta actividad no está en ella misma, sino que sirve única y exclusivamente para conseguir aspectos externos a ella. 
Se está asumiendo, como dice Devís (2001), que la infancia es única y exclusivamente una preparación para la vida adulta y que se le está dando más importancia a aspectos externos a la propia actividad que a los aspectos derivados de su experiencia.

Ante esta situación, fue Arnold (1991) quien propuso una nueva conceptualización para el área que permitiera revalorizarla por ella misma y no por lo que se podría conseguir a través de ella. Cuando en una actividad importa tanto su realización como la manera de practicarla se está entendiendo que fines y medios no están separados sino que ambos son intrínsecamente valiosos.

En este punto es posible comprender la afirmación de Arnold (1991: 23) al decir que: "tanto la materia como el método se convierten en fines y que el método no se le considera simplemente como un medio de promover la materia”. Su propuesta de conceptualización vendría dada por tres dimensiones que delimitarían tres funciones propias de la educación física escolar: 'educación sobre el movimiento', 'educación a través del movimiento' y 'educación en movimiento'.

La primera dimensión atiende a una dimensión intrínseca y se refiere a los conocimientos teóricos relativos al campo de la educación física. La segunda es de carácter instrumental (extrínseco), es decir, contiene aquellos aspectos que podríamos desarrollar a partir del movimiento, asociados a él. Finalmente, la última dimensión tiene un carácter totalmente intrínseco pues se refiere al hecho de que al realizar educación física ésta posee valores propios como el autoconocimiento de quien la practica.

Arnold (1991) plantea que las funciones verdaderamente educativas son aquellas ligadas al carácter intrínseco de la educación física, es decir, a las dimensiones 'sobre' y 'en'. Sin duda que, aún considerando el importante aporte de los propósitos extrínsecos en la revalorización de la educación física escolar, podríamos decir que ésta sigue estando sustentada en una visión epistemológica dualista que separa y que sigue entendiendo que cuerpo y cognición son dos realidades paralelas, idea muy alejada de la nueva dimensión del ser humano (Damasio, 2003; Morín, 2003; Varela, 2000). Además, tal propuesta "se elabora dentro de un vacío sociocultural que no entra a valorar factores y condicionantes sociales y contextuales que rodean a la educación física" (...) y que, por tanto, podría terminar "reproduciendo el status quo imperante" (Devís y Molina, 2004: 39)

Los cambios sociales acaecidos en los últimos tiempos han provocado el paso de la modernidad a la llamada sociedad postmoderna. Este cambio ha originado que a la escuela, como institución social, se le exijan una serie de funciones a cumplir. Y, por extensión, la educación física como asignatura del curriculum escolar debe ajustarse a dichos requerimientos. Se observa una traslación de las "culturas de la certeza a las culturas de la incertidumbre" (Hargreaves, 1996: 85), y esto provoca ciertos cambios en la institución escolar, el currículum y en las funciones de la educación física como asignatura del currículum escolar.

Según Devís y Molina (2004: 40), estos cambios asociados a la sociedad postmoderna provocan que la escuela y el currículo creados para la reproducción material y cultural en la modernidad se orienten, ahora, hacia una escuela comprometida con procesos de transformación basados en la justicia social y en la reconstrucción del conocimiento.

En función de lo anteriormente citado, la escuela y, por extensión, la educación física se orientarán hacia una serie de funciones sociales y otras educativas. Siguiento, nuevamente, a Devís y Molina (2004), podemos sintetizar las funciones de la educación física, en la época postmoderna, como sigue a continuación. 
La primera función dice relación con la 'justicia social', es decir, con que la escuela tiene como uno de sus objetivos prioritarios la 'compensación de las desigualdades' de origen (Pérez, 1993). En nuestra área esto quedaría reflejado en orientar nuestras acciones hacia la promoción de valores y actitudes tales como el respeto a las personas y la igualdad. Sería, también, convertir la educación física en una asignatura que sea capaz de favorecer prácticas positivas y beneficiosas de actividad física para los más necesitados de los beneficios de éstas.

"Plantear el problema de la diversidad y de la diferencia en y ante la educación supone enfrentarse con retos y ámbitos de signficados muy polivantes: la lucha contra las desigualdades, el problema de la escuela única interclasista, la crisis de los valores y del conocimiento tenidos por universales, las respuestas ante la multiculturalidad y la integración de minorías..." (Gimeno, 2000: 69).

En cuanto a las funciones educativas de la escuela y de la educación física, éstas tienen que ver con la 'reconstrucción de los conocimientos y las experiencias' que el alumnado tiene en su vida paralela y anterior a la escuela (Pérez, 1993; 1998). Según Devís y Molina (2004: 42) "en el ámbito de la educación física, la reconstrucción se refiere al conocimiento teórico y al conocimiento práctico o experiencial..."

"Si en la vida cotidiana el individuo aprende reinterpretando los significados de la cultura, mediante continuos y complejos procesos de negociación, también en la vida académica, el alumno/a debería aprender reinterpretando y no sólo adquiriendo la cultura elaborada en las disciplinas académicas, mediante procesos de intercambio y negociación. El aula debe convertirse en un foro abierto de debate y negociación de concepciones y representaciones de la realidad. No puede ser nunca un espacio de imposición de la cultura, por más que ésta haya demostrado la potencialidad virtual de sus esquemas y concepciones" (Pérez, 1993: 73).

\section{CONCLUSIONES: UNA PUERTA DE ENTRADA AL PRESENTE MONOGRÁFICO}

Es desde esta clasificación de las funciones de la EFE y, más concretamente, desde las funciones relacionadas con el trabajo pedagógico en pos de mayores niveles de justicia social y de prácticas educativas focalizadas en la reconstrucción del conocimiento paralelo y anterior a la experiencia escolar (Devís y Molina, 2004; Moreno, 2011), que creemos que el monográfico que a continuación se presenta puede ser un verdadero aporte en la difusión de miradas, perspectivas, investigaciones y prácticas educativa-escolares, en el ámbito de la EFE, que alumbren a maestros, profesores y académicos del contexto nacional e internacional para pensar y repensar prácticas educativas e investigativas que propendan a la transformación de esta desigual sociedad.

\section{REFERENCIAS BIBLIOGRÁFICAS}

Almonacid, A. Matus, M. (2010). The emergence of imagination of student in school from the hall of physical education. Fiep Bulletin, Vol. 80, $758-761$.

Arnold, P. (1979). Educación Física, movimiento y currículum. Madrid: Morata. 
Barbero, J. (2007). Capital(es) corporal(es) que configuran las corrientes y/o contenidos de la educación física escolar. En Ágora para la Educación Física y el Deporte, Vol.4-5, 21-38.

Benjumea, M. (2009). Elementos constitutivos de la motricidad como dimensión humana. Tesis para optar al grado de Magister en Motricidad y Desarrollo Humano. Medellín: Instituto de Educación Física Universidad de Antioquia.

Damasio, A. (2003). En busca de Spinoza. Neurobiología de la emoción y los sentimientos. Barcelona: Editorial Crítica.

Devís, J. y Molina, J. (1998a). Funciones de la educación física en el currículum escolar. En A. García, F. Ruiz y A. Casimiro (coord.), La enseñanza de la Educación Física y el Deporte escolar. Actas del II Congreso Internacional (pp.48-51), Málaga: I.A.D.-Junta de Andalucía.

Devís, J. y Molina, J. (1998b). Educación física escolar: funciones, racionalidad práctica e ideología. En M. Villamón (dir.), La Educación Física en el currículum de Primaria (pp.13-32). València: Consellería de Cultura, Educació i Ciència de la Generalitat Valenciana.

Devís, J. y Molina, J. (2004). Las funciones de la educación física escolar: de la modernidad a la postmodernidad. En F. Caparroz, y N. De Andrade (Dir.), Educaçao física escolar. Política, investigaçao e intervençao (pp.35-49). Brasil: LESEF/UFFS y NEPECC/UFU.

Devís, J. (1994) Educación física y desarrollo del currículum: un estudio de casos en investigación colaborativa. Tesis doctoral. València: Universitat de València.

Devís, J. (2001). La educación física, el deporte y la salud en el siglo XXI. Alicante: Editorial Marfil.

Díaz Lucea, J. (1994). El currículum de la Educación física en la reforma educativa. Barcelona: Inde.

Gimeno, P. (2009). Didáctica crítica y comunicación. Un diálogo con Habermas y la Escuela de Frankfurt. Barcelona: Octaedro.

Habermas, J. (1999). Teoría de la acción comunicativa. Madrid: Taurus.

Hargreaves, A. (1996). Profesorado, cultura y postmodernidad. Cambian los tiempos, cambia el profesorado. Madrid: Editorial Morata.

Hernández, J. (1996). La construcción histórica y social de la educación física: el currículo de la LOGSE, ¿una nueva definición de la educación física escolar? En Revista de Educación, n. $311,51-76$

Jaramillo, L. (2010). La Motricidad Humana como núcleo fundante al interior del grupo Kon-moción. En Nervaduras de la Motricidad Humana (pp. 55-70). Popayán: Universidad del Cauca..

Le Boulch, J. (1969). La educación por el movimiento. Buenos Aires: Paidos.

Martín, F. (2009). Competencias Básicas y Funciones de la Educación Física en la LOE. En Revista Digital Innovación y Experiencias Educativas, 16 www.csi-csif.es/.../FRANCISCO \%20JESUS_MARTIN_1.pdf [Web visitada 30 marzo 2010 a las 11.16 horas].

MINEDUC (2009). SIMCE nacional. En http://www.simce.cl/ [Web visitada 23 julio 2010 a las 10.34].

MINEDUC (2010). Planes y programas de estudio. En www.mineduc.cl [Web visitada 14 enero 2010 a las 18.43 horas].

MINEDUC. Decreto 220/2005 por el que se establece los objetivos fundamentales y contenidos mínimos obligatorios para enseñanza media y fija normas para su aplicación. Santiago de Chile: Gobierno de Chile.

Moreno, A. (2011). Percepciones del profesorado universitario en relación a la función de transformación de la educación física como asignatura de curriculum escolar: el caso de Chile. Tesis Doctoral Inédita. Granada: Universidad de Granada.

Morin, E. (2001). Introducción al pensamiento complejo. Barcelona: Gedisa.

Morin, E. (2003). El método. La humanidad de la humanidad. Madrid: Cátedra.

Pérez, A. (1993) Las funciones sociales de la escuela: de la reproducción a la reconstrucción crítica del conocimiento y la experiencia. En J. Gimeno y A. Pérez, Comprender y transformar la enseñanza (pp. 17-33). Madrid: Morata.

Pérez, A. (1998). La cultura escolar en la sociedad neoliberal. Madrid: Morata. 
Romero, C. (s/f). Evolución y desarrollo de las funciones atribuidas al movimiento como elementos formativo. www.ugr.es/...\%20cuerpo $\% 20$ y\%20movimiento/funciones\%20EF.pdf [Web visitada 14 de agosto Sergio, 2009]

Sergio, M. (2006). Motricidad Humana ¿Cuál es el futuro? En Revista pensamiento educativo Vol. 38, $14-33$

Sergio, M. (2009). Críticas a la Ciencia de la Motricidad Humana. En M. Sergio et al. (2009). Motricidad Humana: una mirada retrospectiva (pp.31-56). España-Colombia: Instituto Internacional del Saber.

Toro, S. (2007a). Una aproximación epistemológica a la didáctica de la motricidad desde el discurso y práctica docente. Revista Estudios Pedagógicos, XXXIII, n.1, 29-43.

Toro, S. (2007b). Memoria y evolución. Pilares de una educación consciente desde la motricidad. Revista Online Profissao Docente, 16. http://www.uniube.br/propep/mestrado/revista/artigos. php\# Web visitada 13 julio 2009 a las 9.17 horas.

Toro, S. (2009). Conocimiento desde una mirada latina o crítica. En M. Sergio et al. (2009). Motricidad Humana: una mirada retrospectiva (pp.107-127). España-Colombia: Instituto Internacional del Saber

Trigo, E. y Montoya, H. (2004). Aportes de la Ciencia de la Motricidad Humana a la Educación Física, Recreación y Deporte. Universidad del Cauca. Colombia. Disponible en http://www. kon-traste.com/pdf/articulos_desde_2004/aportes.pdf

Trigo, E. (2006). Inteligencia creadora, ludismo y motricidad. En Acción 3 Consentido. Popayán: Universidad del Cauca.

Trigo, E. y Montoya, H. (2007). Aportes de la Motricidad Humana a la Educación Física. En Motricidad y Persona, n.3, 9-43.

Varela, F. (2000). El fenómeno de la vida. Santiago de Chile: Dolmen.

Vázquez, V. (2001). Los fundamentos de la Educación Física. En V. Vázquez (Coord.), Bases Educativas de la Actividad Física y el Deporte (pp.47-68). Madrid: Editorial Síntesis.

Zubiri, X. (1986). Sobre la existencia. Madrid: Alianza. 